Review

International Journal of Biological Sciences

ISSN 1449-2288 www.biolsci.org 2008 4(3):126-132

CIvyspring International Publisher. All rights reserved

\title{
Sex Hormones' Regulation of Rodent Physical Activity: A Review
}

\section{J. Timothy Lightfoot}

Dept. of Kinesiology, University of North Carolina Charlotte, Charlotte, NC 28223, USA

Correspondence to: J. Timothy Lightfoot, Belk Gym, Dept. of Kinesiology, UNC Charlotte, Charlotte, NC 28223. 704-687-4692 (v); 704-687-3350 (fax); jtlightf@uncc.edu

Received: 2008.03.12; Accepted: 2008.04.24; Published: 2008.04.29

There is a large body of emerging literature suggesting that physical activity is regulated to a varying extent by biological factors. Available animal data strongly suggests that there is a differential regulation of physical activity by sex and that the majority of this differential regulation is mediated by estrogen/testosterone pathways with females in many animal species having higher daily activity levels than males. The purpose of this manuscript is to review the mechanisms by which estrogen, progesterone, and testosterone affect the regulation of physical daily activity. This review lays the foundation for future investigations in humans as well as discussions about relative disease risk mediated by differential biological regulation of physical activity by sex.

Key words: estrogen, testosterone, progesterone, mammals, mice, physiology

\section{Introduction}

The use of animal models as proctors for human physiology has a long and significant history. Subsequently, some researchers have turned to using animal models to increase understanding of the mechanistic underpinnings of exercise behaviors. This work is critical given the literature that indicates a general decline in physical activity [50] and an increase in obesity and other hypokinetic diseases in most Western-cultures [27]. While physical activity has normally been considered a 'voluntary activity', held to free-will and influenced solely by environmental factors there is a growing body of literature that suggests that physical activity is significantly regulated by biological factors. These biological regulating factors may take many forms including a central nervous center-located "activity-stat" [38], an increase or decrease in various physiological substance or structures (e.g. GLUT4 ref. [51]), and/or genetics [23, 25, 46]. Biologically, the sex hormones play a large role in regulating various physiological parameters in both males and females and thus would be the natural subject of investigations into possible roles they play in regulating physical activity. Therefore, the focus of this paper is to review the literature investigating the effect of and the physiological mechanisms through which estrogen, progesterone, and testosterone may regulate physical activity. Given the sparseness of the human literature in this area, the primary model used in this research has been animal models.

\section{Sex differences in physical activity}

In general, it is commonly accepted that human females are less active than males; this sex differentiation in activity is prevalent in both children and adolescents [31] as well as adults [50]. However, confounding factors in human research in this area, including the well-known difficulty of accurately measuring physical activity in large populations [42], the complexity of giving hormone treatments in a physiologically-relevant and ethical manner [52], the general lack of well-controlled prospective human studies in this area, and the near impossibility of controlling environmental variations that can influence activity levels make understanding human sex differences in activity extremely problematic. Given these difficulties and the relative ease of experimental control in animal studies, it is appropriate to consider whether there is a sex difference in activity present in animal models.

The rodent literature that addresses sex hormone effects on activity generally shows that female rodents are more active than male rodents. Figure 1 is a summary of representative literature that has observed sex differences in daily activity in rats or mice. Overall, female rodent activity levels on a daily basis range from $20 \%$ to over $50 \%$ higher than males. Bronstein, et al. [6] observed that female Sprague-Dawley rats traversed significantly more distance during open-field testing (ranging from $28-46 \%$ more) than male rats, regardless of whether the animals had been handled or not. Craft, et al. [8] while considering sex differences in morphine-induced locomotor changes, observed that in the baseline state, female Sprague-Dawley rats exhibited $46 \%-72 \%$ more activity (as measured by photobeam breaks in their home cage) than males over a 3-5 hour period, depending upon 
whether they had been handled or not. Li, et al. [24] showed that there may be strain differences in these sex-related patterns of activity by showing that while the total path length that Wistar and WKY rats ran were significantly higher in females, there were no observed differences between sexes in the SHR rats. An earlier strain screen study in mice [25] showed that while overall female mice ran approximately $20 \%$ further per day than male mice, there was large variability in this percentage depending upon which of the 13 strains were tested. The percentage difference between female and male mice of the same strain in distance run on a daily basis ranged from $-21.9 \%$ (SPRET/EiJ) to $111.2 \%(\mathrm{C} 3 \mathrm{H} / \mathrm{HeJ})$. Similar variation was also observed in duration and speed phenotypes. This type of between strain variation in photobeam break activity data has also been observed by Seburn within the Mouse Phenome database [41]. Earlier work by Koteja, et al. [21] monitored wheel running activity in both control Hsd:ICR mice and mice from generation 10 of an ongoing study to selectively breed mice for wheel-running activity. While there were significant differences between the control and selected lines in daily wheel-running activity, there were also significant sex-differences in the amount of distance run reported in both the control lines $(50 \%$ higher in females) and the selected lines (58\% higher in females). Recent work from our laboratory [26] with a large cohort of $F_{2}$ animals $(n=310)$ developed from reciprocally breeding high active $\mathrm{C} 57 \mathrm{~L} / \mathrm{J}$ and low active $\mathrm{C} 3 \mathrm{H} / \mathrm{HeJ}$ inbred mouse strains have shown that on average (over 21 days), the female animals ran $47 \%$ farther, 39\% longer, and 9\% faster than male animals.

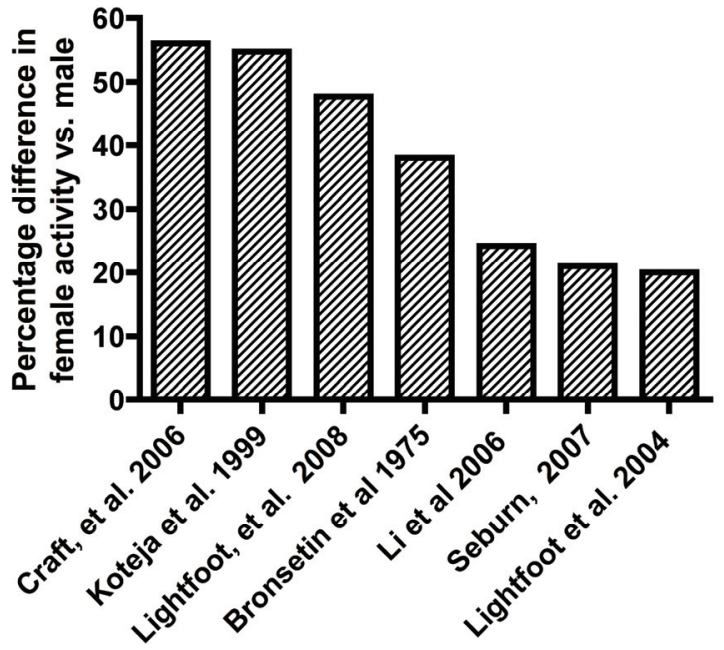

Figure 1 - Representative literature reporting percentage difference in female activity vs. male activity in rodents.

In summary, the majority of the animal literature over the past 80 years, have largely accepted that physical activity can be governed by biological factors and thus, activity levels are routinely treated as the dependent variable in many study designs. Additionally, given that most environmental factors can be controlled when using animal models, the animal literature has appropriately focused upon the role that sex hormones play in regulating daily activity, as Gorzek, et al. [15] did in investigating the effect of estrogen on daily activity levels.

\section{Effects of sex hormones on activity in ani- mal models}

The investigation of the effect of sex hormones on physical activity in rodent models has a long and varied history. Figure 2 (R. Bowen personal communication) shows the interesting waxing and waning of such research over the past 80 years. While a complete historical review of this topic is outside the scope of this paper, it is of note that the consideration of sex hormone effects on activity was initiated in the early 1920 's when it was found that ovariectomies significantly decreased activity in female rats in conjunction with the observation of a marked decrease in wheel-running activity in male rats after castration $[18,35,44]$. These studies also noted that implantation of ovarian tissue into both female rats [35, 44] and male rats [54] 'recovered' the pre-neutering activity patterns whereas implantation of testes into both male and female rats increased activity to a lesser extent than the ovarian implants [36]. The evidence from these studies, produced before estrogen, progesterone, or testosterone were isolated and identified, were so strong that one researcher concluded that in female rats, "Obviously, therefore, the spontaneous activity is dependent on ovarian function". However, this same researcher cautioned that none of the available experiments proved that the "hormone actually produces activity" (their emphasis) [35].

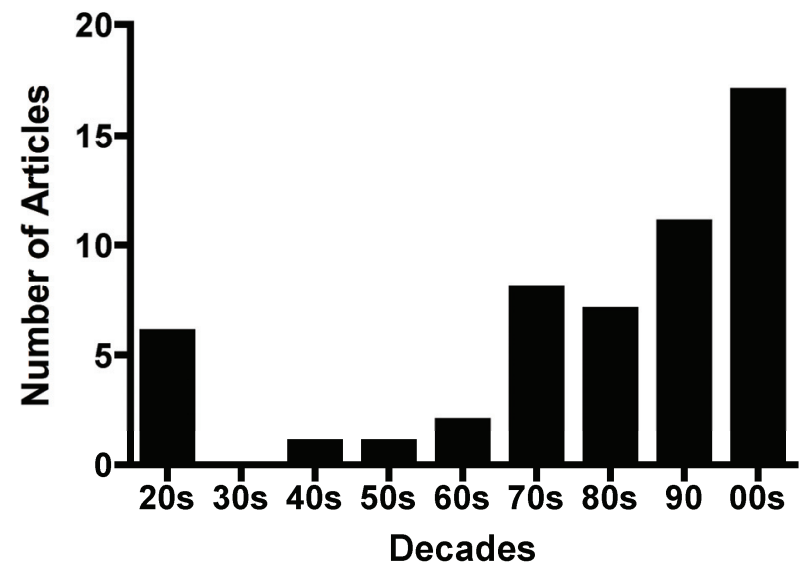

Figure 2 - The yearly distribution of studies regarding the effect of sex hormones on physical activity. The x-axis designates the ten-year period in which the studies were published. (Personal communication from R. Bowen) 
Several studies since these early findings have shown that estrogen and testosterone directly impact spontaneous activity in rodent models. For example, recently Gorzek and colleagues [15] showed that while ovariectomies reduced daily wheel-running activity in female mice, a dosage of $3 \mu \mathrm{g} \bullet \mathrm{d}^{-1}$ of $17 \beta$-estradiol increased activity to a level comparable to activity in sham-operated mice. Roy and Wade [39] demonstrated that administration of varying doses of testosterone propionate $(100 \mu \mathrm{g}-1 \mathrm{mg} /$ day $)$ increased running wheel activity in castrated male rats. These two studies, which are two examples of numerous studies, provide evidence of a cause/effect relationship between reproductive hormones and spontaneous activity in rats and mice.

\section{Estrogen}

While several studies have observed a causal relationship between the sex hormones, particularly estrogen, and spontaneous activity, the mechanistic underpinnings of this relationship has been somewhat unclear. Cushing, in a series of experiments (summarized by ref. [9]), investigated the role of sexual receptivity on the relationship between estrogen and activity using prairie voles. Female prairie voles, unlike other female rodents, are unique in that they undergo induced estrus by exposure to male voles and their sexual receptivity does not require progesterone, thus, removing two possible confounding factors that might influence activity. Cushing and Hite [9] found that sexually mature virgin female voles did not increase seven-day wheel running behavior either with or without estradiol injections $(0.05 \mu \mathrm{g}$ or $0.5 \mu \mathrm{g}$ estradiol). It had been earlier shown that the number of estradiol receptors in the brain had previously been shown to increase in female voles when exposed to males [7]. Thus, the observation of a the lack of an increase in spontaneous activity with estradiol administration before exposure to males suggested that estradiol's effect on spontaneous activity was linked to the increased number of estradiol receptors in the brain.

Most research investigating the mechanisms of estrogenic increases in activity have focused on central neural pathways $[12,13,30]$. Bilateral implantation of estradiol in the medial preoptic area and the anterior hypothalamus was shown to variably increase wheel running activity in ovariectomized female rats [12]. Since the preoptic area contains both estrogen receptor isoforms - alpha $(E R \alpha)$ and beta $(E R \beta)$ - while the hypothalamic areas contain primarily $\mathrm{ER} \alpha$ and little if any ER $\beta$ [43], Fahrbach, et al. [12] suggested that ER $\alpha$ may control the estrogenergic effect on activity. This hypothesis was difficult to test since $17 \beta$-estradiol has a similar affinity for both ER $\alpha$ and ER $\beta$ receptor subtypes [22].

Coumesterol is a phytoestrogen with similar affinity as $17 \beta$-estradiol for ER $\beta$, but four-fold less affinity for ER $\alpha$ and seven-fold higher affinity for ER $\beta$ than for ER $\alpha$ [22]. Garey and colleagues [13] injected coumestrol $(10 \mu \mathrm{g})$, singly and in combination with estradiol implants $(2.5 \mu \mathrm{g})$ in 48 adult, ovariectomized Swiss-Webster mice and measured running wheel activity for the following 10 days. Running wheel activity increased with estradiol administration (Fig. 3), but coumestrol administration did not alter running wheel behavior as compared to a placebo injection. From this data, the authors suggested that the ER $\beta$-pathway did not influence spontaneous activity; rather, the increases in spontaneous activity arising from estrogen administration were mediated primarily through the ER $\alpha$ pathway. Additionally, Garey, et al.'s data also eased concerns that the phytoestrogens in the standard rodent chows that have been used in many of the published rodent activity papers (e.g. [20, 21, 23, 25, $47,48,53])$ have not confounded the conclusions of these studies as has been noted for other areas of rodent physiology (e.g. [45]).

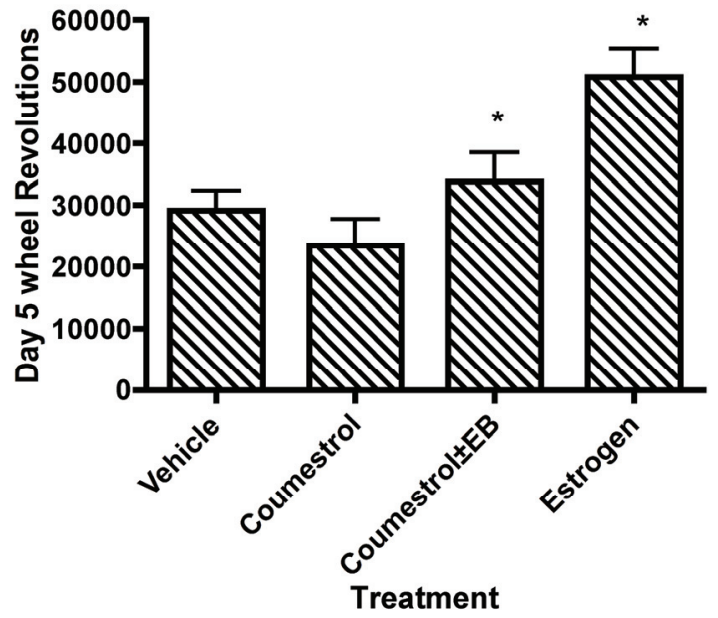

Figure 3 - Wheel running behavior after five days of phytoestrogen supplementation. *Significantly different from vehicle/placebo treatment. Drawn from tabular data presented by Garey, et al. [13].

Garey and colleagues' [13] suggestion that the estrogen-induced increases in spontaneous activity are mediated through the ER $\alpha$ pathway has been supported by further experiments with knock-out animals. Ogawa, et al. [30], using gonadectomized knock-out mice that lacked either ER $\alpha$ or ER $\beta$ genes, implanted either a $17 \beta$-estradiol capsule (16 or 160 $\mathrm{ng} / \mathrm{d}$ ) or a placebo capsule and monitored running wheel activity for nine days. Ogawa and colleagues observed that both male and female wild-type mice (i.e. with $E R \alpha$ and $E R \beta$ pathways intact) increased 
running wheel activity significantly with either 16 or $160 \mathrm{ng} / \mathrm{d}$ estradiol treatments as compared to placebo mice. Additionally, with estradiol treatments, both sexes of the ER $\beta$ knock-out mice also significantly increased their running wheel activity compared to placebo; however, even with estradiol administration, the $\mathrm{ER} \alpha$ knock-out mice did not increase activity compared to placebo. These results, in conjunction with Garey, et al.'s earlier study on coumesterol [13] lend strong evidence to the hypothesis that the estrogenic effects on running wheel behavior in mice are mediated through the ER $\alpha$-pathway.

While it appears that estrogenic activation of the ER $\alpha$-pathway is the primary mediator of the increase in running wheel behavior, the mechanisms through which the ER $\alpha$-pathway influences activity are unclear. Morgan, et al. [28] have speculated that the ER $\alpha$-pathway modulates several neurotransmitters, including dopamine and/or serotonin, which may play a role in the regulation of activity. In a review of the interactions of the sex differences in dopaminergic regulation, Becker [3] proposed that in female rats, estrogen enhanced terminal release of dopamine relative to male rats, which explains, the authors note, many reproductive-related behaviors such as increased pacing behavior. Additionally, it has been observed that estrogen can regulate several different steps in dopaminergic functioning, including dopamine release and metabolism as well as the pre- and post-synaptic receptor and transporter functioning (for review see [10]). Interestingly, effects of estrogen on dopamine receptors and transporter mechanisms in the nucleus accumbens have been noted to be as fast as two minutes; faster than would be expected from genomic activation [49] suggesting an influence of estrogen on the dopaminergic systems not arising through activation of either the ER $\alpha$ or ER $\beta$ pathways. Supporting this nongenomic activation hypothesis are observations of nongenomic effects of estrogens postulated to occur through the activation of a common membrane binding site with the same pharmacological profile as the hypothesized $\gamma$-adrenergic receptor [29].

Whether a differential activation of the dopaminergic systems by the ER $\alpha$ pathway or some other nongenomic pathway leads to the sex-related difference in activity levels is currently unknown. However, the involvement of dopamine in regulating daily activity is supported by indirect evidence. Garland's group [33, 34] have suggested that an alteration in dopaminergic functioning is one of the primary characteristics of mice experimentally evolved for high activity. Specifically, Rhodes and Garland have observed that an inhibition of dopamine transporter
(DAT) will reduce the wheel-running of mice that are high active [34]. Additionally, they have shown that female high active mice have a reduced functioning of the D1 and D5 dopamine receptors [33]. Whereas Rhodes and Garland only studied female mice in each of their studies, it is yet to be determined if these alterations in dopaminergic functioning are different in male high active mice. Thus, while tenuous evidence suggests that the estrogen activation of the ER $\alpha$-pathway and a possible nongenomic pathway may lead to an enhanced release of dopamine in female rodents which coupled with alterations in dopamine transport and/or receptors, may lead to increased activity, it is unclear whether this results in differential levels of activity between sexes.

\section{Progesterone}

While there have been numerous investigations of estrogen's role in determining physical activity levels in animal models, there have been virtually no studies considering whether progesterone influences activity. Progesterone is known to impact several physiological mechanisms and given that estrogen and progesterone normally cycle together during at least one portion of the female reproductive cycle [16], progesterone could also affect activity behavior. In a larger study concentrating on the differential effect of estradiol and estrone on activity, Young and Fish [55] noted that progesterone injected in conjunction with estradiol did not influence activity in rats in any manner. This result was expanded upon in a three-phase study conducted by Rodier [37] which is the only other currently available study in this area. Sherman albino rats received varying dosages of progesterone $(4,8$, or $40 \mathrm{mg} / \mathrm{kg} /$ day $)$ or placebo injections. Rodier noted that injections of 8 or 40 $\mathrm{mg} / \mathrm{kg} /$ day in gonadally-intact rats decreased normal wheel-running activity, but neither an injection of 4 $\mathrm{mg} / \mathrm{kg}$ /day progesterone or placebo solvents changed normal activity levels. Rodier then ovariectomized seven of the rats, injected them with 40 $\mathrm{mg} / \mathrm{kg} /$ day progesterone for six days and then injected them with solvent for another six days. After ovariectomy, activity decreased to a level similar to that seen with progesterone injection in intact animals and was not further depressed with subsequent progesterone injection suggesting that progesterone was interfering with endogenous estrogens' effects on physical activity. To determine if exogenous estrogen interacted with the progesterone inhibition of wheel-running activity, Rodier then injected $17 \beta$-estradiol $(100 \mu \mathrm{g} / \mathrm{kg})$ into the ovarectomized and control animals, with each injection separated by five days. Eight days after the last estradiol injection, he injected either progesterone $(40 \mathrm{mg} / \mathrm{kg} /$ day $)$ or a pla- 
cebo. While the estrogen injections increased the animals' running wheel behavior for at least 21 days after the first injection, the animals receiving the progesterone injections sharply decreased their activity, which then increased again after the cessation of the progesterone injections. Thus, given that either intrinsic or extrinsic estrogen had to be present for the inhibitory effects of progesterone to be observed, Rodier suggested that the progesterone decrease in physical activity was mediated through a direct interference with estrogen. A negative interference of progesterone on estrogenergic effects on neurotransmitters has been observed in both rats [17] and primates [14]; conversely, there is also literature available suggesting that progesterone augments [4] or has no effect [2] on estrogenic effects on neurotransmitters. However, an interference of progesterone on estrogen's physical activity effects is attractive to hypothesize because such an interference would explain the common sinusoidal activity pattern observed in female rats [35]; it could be suggested that peak activity was occurring during the follicular phase due to the primacy of the estrogen peak with the subsequent decrease in physical activity occurring during the luteal phase when progesterone is the dominant hormone and is interfering with estrogen's activity increasing effects. The dearth of investigations in this area leaves this and other hypotheses related to progesterone and estrogen's interactions on activity, such as the role of physiological doses of progesterone in activity regulation and the specific site(s) and mechanism(s) of the estrogen/progesterone interference open for further investigation.

\section{Testosterone}

In rodents, several studies using orchidectomized males have indicated a potential role for sex hormones in the regulation of activity $[5,36,39]$. For example, Broida and Svare [5] showed that locomotor activity - measured by photobeam breaks - could be rescued in castrated animals by implanting silastic capsules containing testosterone. Additionally, Broida and Svare suggested that there may be a genotype dependency in these responses, since they observed a larger decrement in physical activity after castration in inbred C57BL/6J mice as compared to inbred DBA/2J and random-bred Rockland-Swiss mice [5].

While a possible role for testosterone in the regulation of daily activity has been proposed $[5,36$, 39], few studies have attempted to determine mechanistic causes of this effect. Roy and Wade [39] in a widely quoted study, injected either testosterone (100 $\mu \mathrm{g} /$ day), estradiol (10 $\mu \mathrm{g} /$ day), dihydrotestosterone propionate (DHTP - $100 \mu \mathrm{g} /$ day), or sesame oil (placebo) into 20 castrated adult male Sprague-Dawley rats and then monitored running wheel activity for 16 days. They noted that testosterone injections significantly increased the running wheel activity of the rats $(p<0.05)$ but not as much as estradiol injections $(p<0.02)$. Neither the DHTP or placebo injections increased running activity above baseline. Given that testosterone is aromatizable to estrogen whereas DHTP is not, the authors concluded that the primary mechanism regulating running wheel activity with testosterone administration was the aromatization of the testosterone into estrogen. In the second part of this experiment [39], these authors found that administration of an estrogen antagonist (MER-25) attenuated the increased running wheel activity resulting from either estradiol or testosterone injection. These results further supported the hypothesis that the increased activity from testosterone injection was mediated by the estrogen pathways.

While it appears that testosterone injections/implants can rescue activity levels after orchidectomy, supplementation of testosterone in intact animals does not increase physical activity levels. Eleftheriou and colleagues observed that testosterone level was not significantly correlated to a single 24 hour observation of wheel-running activity [11] in gonadally intact male C57BL/6By and Balb/cBy mice, in seven recombinent inbred strains (CXBD, CXBE, CXBG, CXBH, CXBI, CXBJ, and CXBK) or in two $\mathrm{F}_{1}$ hybrid strains $\left(\mathrm{B}^{6} \mathrm{CF}_{1}\right.$ and $\left.\mathrm{CB} \mathrm{F}_{1}\right)$. Salvador, et al. [40] showed that suprapharmacological doses $(3.75,7.5,15$, or $30 \mathrm{mg} / \mathrm{kg}$ ) of artificial anabolic-androgenic steroids (testosterone propionate and nandrolone decanoate), injected into gonadally intact male OF1 male mice, did not affect running wheel activity measured 24 hours after the injection. Furthermore, mice injected with suprapharmacological doses for 10 weeks, did not show alterations in activity levels compared to baseline during 24 hour monitoring periods in the eight, ninth, or tenth week. These results support work in rats by Li and Huang [24] that showed that testosterone injections in both male and female gonadally-intact animals did not generally result in an increase in total path length. Thus, while work in gonadally-deficient animals has shown that testosterone, after aromatization to estrogen, will increase physical activity levels in rodents, supplementation above baseline levels will not further increase activity.

\section{Summary}

A wide-variety of evidence suggests that in rodents, females are generally more active than males, with a large proportion of this differential activity explained by sex hormone effects. The mechanism of this regulation (Fig. 4) appears to be mediated through the 
estrogen- $\alpha$ receptor pathway with the requirement of aromatization of testosterone to estrogen in males. Little literature exists to explain the mechanism leading from estrogen- $\alpha$ receptor activation to an increase in activity; however, recent literature has suggested that alterations in dopaminergic systems as well as possible non-genomic actions of estrogen may be involved. The few available human studies in this area $[1,19,32]$ have contained multiple confounders that make deriving any useful conclusions from these studies difficult at best. Thus, extensions of the existing mechanistic studies on ER $\alpha$-pathway regulation of physical activity in rodents as well as more carefully controlled human studies are needed to determine if sex hormones provide any physiological regulation of activity in humans and if so, possible pathways through which this effect may be mediated. The increasing rate of inactivity which has led to the increased cardiovascular and other hypokinetic diseases in most populations [27] make understanding the mechanisms of physical activity regulation critical in the context of the health-related goals of our society.

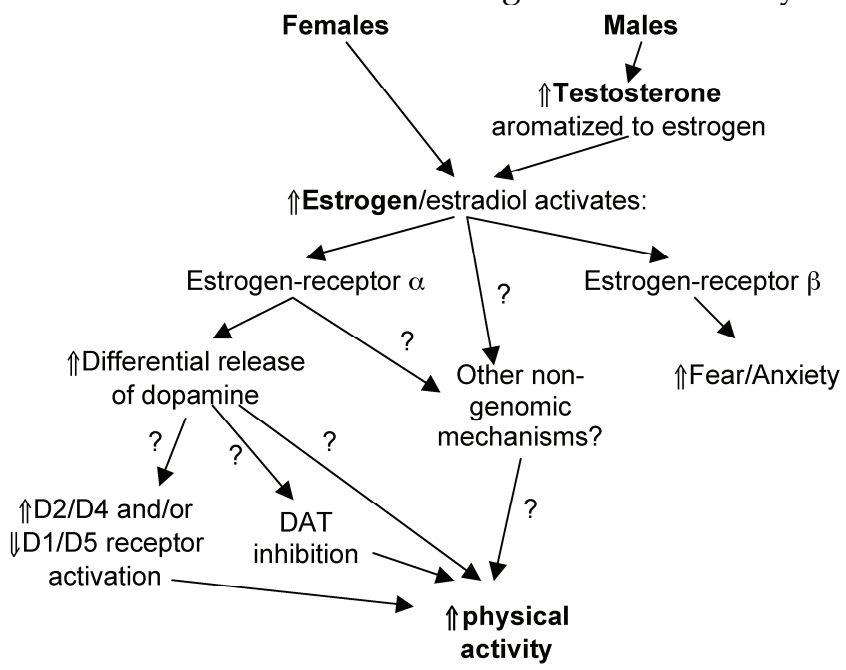

Figure 4 - Hypothesized schematic of the regulation of physical activity by sex hormones in rodents. "?" = pathway currently supported by speculation and/or tentative data. DAT = dopamine transporter; D2/D4, D1/D5 signify different dopamine receptor populations.

\section{Acknowledgements}

The author would like to thank the editing and proof-reading comments of the Kinesiology Writing Accountability Klatch: Drs. M. Turner, T. Hubbard, S. Tsivitse, and M. Cordova as well as suggestions for studies and verbiage to include in this review by $R$. Bowen and A. Knab. This review supported by funding from NIH NIAMS RO1AR050085.

\section{Conflict of interest}

The author has declared that no conflict of interest exists.

\section{References}

1. Anderson EJ, Lavoie HB, Strauss CC, Hubbard JL, Sharpless JL, Hall JE. Body composition and energy balance: lack of effect of short-term hormone replacement in postmenopausal women. Metabolism. 2001; 50: 265-269.

2. Attali G, Weizman A, Gil-Ad I, Rehavi M. Opposite modulatory effects of ovarian hormones on rat brain dopamine and serotonin transporters. Brain Research. 1997; 756: 153-159.

3. Becker JB. Gender differences in dopaminergic function in striatum and nucleaus accumbens. Pharmacology, Biochemistry and Behavior. 1999; 64: 803-812.

4. Becker JB, Rudick C. Rapid effects of estrogen or progesterone on the amphetamine-induced increase in striatal dopamine are enhanced by estrogen priming: a microdialysis study. Pharmacology, Biochemistry and Behavior. 1999; 64: 53-57.

5. Broida J, Svare B. Genotype Modulates Testosterone-Dependent Activity And Reactivity In Male-Mice. Hormones And Behavior. 1983; 17: 76-85.

6. Bronstein PM, Wolkoff FD, Levine MJ. Sex-related differences in rats' open-field activity. Behavioral Biology. 1975; 13: 133-138.

7. Cohen-Parson $M$, Carter C. Males increase serum estrogen and estrogen receptor binding in brains of female voles. Physiol Zool. 1987; 39: 309-314.

8. Craft RM, Clark JL, Hart SP, Pinckney MK. Sex differences in locomotor effects of morphine in the rat. Pharmacology, Biochemistry and Behavior. 2006; 85: 850-858.

9. Cushing BS, Hite R. Effects of estradiol on sexual receptivity, wheel-running behavior, and vaginal estrus in virgin prairie voles. Physiology \& Behavior. 1996; 60: 829-832.

10. Di Paolo T. Modulation of brain dopamine transmission by sex steroids. Rev Neurosci. 1994; 5: 27-41.

11. Eleftheriou BE, Elias MF, Cherry C, Lucas LA. Relationship of wheel-running activity to post-wheel running plasma testosterone and corticosterone levels: a behavior-genetic analysis. Physiology and Behavior. 1976; 16: 431-438.

12. Fahrbach S, Meisel R, Pfaff D. Preoptic implants of estradiol increase whell running but not the open field activity of female rats. Physiol Behav. 1985; 35: 985-992.

13. Garey J, Morgan MA, Frohlich J, McEwen BS, Pfaff DW. Effects of the phytoestrogen coumestrol on locomotor and fear-related behaviors in femal mice. Hormones and Behavior. 2001; 40: 65-76.

14. Gomez-Mancilla B, Bedard P. Effect of estrogen and progesterone on L-dopa induced dyskinesia in MPTP-treated monkeys. Neuroscience Letters. 1992; 135: 129-132.

15. Gorzek JF, Hendrickson KC, Forstner JP, et al. Estradio and tamoxifen reverse ovariectomy-induced physical inactivity in mice. Med Sci Sports Exerc. 2007; 39: 248-257.

16. Guyton AC, Hall JE. Textbook of Medical Physiology, Tenth Edition. Philadelphia: WB Saunders Co. 2000:916-943

17. Hernandez M, Fernandez-Ruiz J, de Miguel R, Ramos J. Time-dependent effects of ovarian steroids on tyrosin hypdroxylase activity in the limbic forebrain of femal rats. J Neural Transm Gen Sect. 1991; 83: 77-84.

18. Hoskins R. The effect of castration on voluntary activity. American Journal of Physiology. 1925; 72: 324-330.

19. Kenny AM, Kleppinger A, Wang Y, Prestwood KM. Effects of ultra-low-dose estrogen therapy on muscle and physical function in older women. Journal of the American Geriatric Society. 2005; 53: 1973-1977.

20. Koteja P, Carter PA, Swallow JG, et al. Food wasting by house mice: variation among individuals, families, and genetic lines. 
Physiology and Behavior. 2003; 80: 375-383.

21. Koteja P, Swallow JG, Carter PA, Theodore Garland J. Energy cost of wheel running in house mice: implications for coadaptation of locomotion and energy budgets. Physiological and Biochemical Zoology. 1999; :72.

22. Kuiper G, Lemmen J, Carlsson B, Corton J, Safe S, van der Saag P, van der Burg B, Gustafsson A. Interaction of estrogenic chemicals and phytoestrogens with estrogen receptor B. Endocrinology. 1998; 139: 4252-4263.

23. Lerman I, Harrison BC, Freeman K, Hewett TE, Allen DL, Robbins J, Leinwand LA. Genetic variability in forced and voluntary endurance exercise performance in seven inbred mouse strains. Journal of Applied Physiology. 2002; 92: 2245-2255.

24. Li J-S, Huang YC. Early androgen treatment influences the pattern and amount of locomotion activity differently and sexually differentially in an animal model of ADHD. Behavioural Brain Research 2006; 175: 176-182.

25. Lightfoot JT, Turner MJ, Daves M, Vordermark A, Kleeberger SR. Genetic influence on daily wheel running activity level. Physiological Genomics. 2004; 19: 270-276.

26. Lightfoot JT, Turner MJ, Pomp D, Kleeberger SR, Leamy LJ. Quantitative trait loci (QTL) for physical activity traits in mice. Physiological Genomics. 2008; 32: 401-408.

27. Mokdad AH, Marks JS, Sroup DF, Gerberding JL. Actual causes of death in the United States, 2000. Journal of American Medical Association. 2004; :291.

28. Morgan M, Schulkin J, Pfaff D. Estrogens and non-reproductive behaviors related to activity and fear. Neuroscience and Biobehavioral Reviews 2004; 28: 55-63.

29. Nadal A, Ropero AB, Laribi O, Maillet M, Fuentes E, Soria B. Nongenomic actions of estrogens and xenoestrogens by binding at a plasma membrane receptor unrelated to estrogen receptor a and estrogen receptro B. Proceedings of the National Academy of Sciences. 2000; 97: 11603-11608.

30. Ogawa S, Chan J, Gustafsson J-A, Korach KS, Pfaff DW. Estrogen increases locomotor activity in mice through estrogen receptor a: Specificity of the Type of Activity. Endocrinology. 2003; 144: 230-239.

31. Pate R, Long B, Heath G. Descriptive Epidemiology of Physical Activity in Adolescents. Pediatric Exercise Science. 1994; 6: 434-447.

32. Redberg RF, Nishino M, McElhinney DB, Dae MW, Botvinick $\mathrm{EH}$. Long-term estrogen replacement therapy is associated with improved exercise capacity in postmenopausal women without known coronary artery disease. Am Heart J. 2000; 139: 739-744.

33. Rhodes JS, Garland T. Differential sensitivity to acute administration of Ritalin, apomorphine, SCH 23390, but not raclopride in mice selectively bred for hyperactive wheel-running behavior. Psychopharmacology (Berl). 2003; 167: 242-250.

34. Rhodes JS, Hosack G, Girard I, Kelley A, Mitchell G, Garland T. Differential sensitivity to actue administration of cocaine, GBR 12909, and fluoxetine in mice selectively bred for hyperactive wheel-running behavior. Psychopharmacology (Berl). 2001; 158 : 120-131.

35. Richter CP. Animal behavior and internal drives. The Quarterly Review of Biology. 1927; 2: 307-343.

36. Richter CP, Wislocki G. Activity studies on castrated male and female rate with testicular grafts, in correlation with histological of the grafts. American Journal of Physiology. 1928; 86: 651-660.

37. Rodier WII. Progesterone-estrogen interactions in the control of activity -wheel running in the female rat. Journal of Comparative and Physiological Psychology. 1971; 74: 365-373.

38. Rowland TW. The biological basis of physical activity. Medicine and Science in Sports and Exercise. 1998; 30: 392-299.

39. Roy EJ, Wade GS. Role of estrogens in androgen-induced spontaneous activity in male rats. J of Comparative and Physiological Psychology. 1975; 89: 573-579.
40. Salvador A, Moya-Albiol L, Martinez-Sanchis S, Simon V. Lack of effects of anabolic-androgenic steroids of locomotor activity in intact male mice. Perceptual Motor Skills. 1999; 88: 319-328.

41. Seburn K. Mouse Phenome Database: Metabolic characterization. US: Jackson Laboratory; 2001.

42. Shephard R. Limits to the measurement of habitual physical activity by questionnaires. British Journal of Sports Medicine. 2003; 37: 197-206.

43. Shughrue P, MLane M, Merchenthaler I. Comparative distribution of estrogen receptor-a and -b mRNA in the rat central nervous system. Journal of Comparative Neurology. 1997; 288: 507-525.

44. Slonaker J. The effect of pubescence, oestruation and menopause on the voluntary activity in the albino rat. American Journal of Physiology. 1924; 68: 294-315.

45. Stauffer BL, Konhilas JP, Luczak ED, Leinwand LA. Soy diet worsens heart disease in mice. Journal of Clinical Investigation. 2006; 116: 209-216.

46. Stubbe JH, Boomsma DI, De Geus EJC. Sports participation during adolescence: A shift from environmental to genetic factors. Medicine and Science in Sports and Exercise. 2005; 37: 563-570.

47. Swallow JG, et al. Effects of voluntary activity and genetic selection on aerobic capacity in house mice (Mus domesticus). Journal of Applied Physiology. 1998; 84: 69-76.

48. Swallow JG, Koteja P, Carter PA, Garland T. Artifical selection for increased wheel-running activity in house mice results in decreased body mass at maturity. Journal of Experimental Biology. 1999; 202: 2513-2520.

49. Thompson T, Moss R. Estrogen regulation of dopamine release in the nucleus accumbens: genomic- and nongenomic-mediated effects. J Neurochem. 1994; 62: 1750-1756.

50. Troiano RP, Berrigan D, Dodd KW, Masse LC, Tilert T, McDowell M. Physical Activity in the United States Measured by Accelerometer. Medicine and Science in Sports and Exercise. 2008; 40: 181-188.

51. Tsao T-S, Li J, Change KS, Stenbit AE, Galuska D, Anderson JE, Zierath JR, McCarter RJ, Charron MJ. Metabolic adaptations in skeletal muscle overexpressing GLUT4: effects on muscle and physical activity. FASEB Journal. 2001; 15: 958-969.

52. Turgeon J, Carr M, Maki P, Mendelsohn M, Wise P. Complex actions of sex steroids in adipose tissue, the cardiovascular system, and brain: Insights from basic science and clinical studies. Endocrine Reviews. 2006; 27: 575-605.

53. Turner MJ, Kleeberger SR, Lightfoot JT. Influence of genetic background on daily running-wheel activity differs with aging. Physiologic Genomics. 2005; 19: 270-276.

54. Wang GH, Richter CP, Guttmacher AF. Activity studies on male castrated rats with ovarian transplants, and correlation of the activity with the histology of the grafts. American Journal of Physiology. 1925; 72: 581-599.

55. Young WC, Fish WR. The ovarian hormones and spontaneous running activity in the female rat. Endocrinology. 1945; 36: 181-189. 